\title{
Risk minimizing portfolios and HJBI equations for stochastic differential games
}

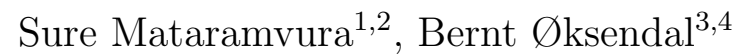

Revised 10 August 2007

\begin{abstract}
In this paper we consider the problem to find a market portfolio that minimizes the convex risk measure of the terminal wealth in a jump diffusion market. We formulate the problem as a two player (zero-sum) stochastic differential game. To help us find a solution, we prove a theorem giving the HJBI conditions for a general zero-sum stochastic differential game in a jump diffusion setting. We then use the theorem to study particular risk minimization problems. Finally, we extend our approach to cover general stochastic differential games (not necessarily zero-sum), and we obtain similar HJBI equations for the Nash equilibria of such games.
\end{abstract}

Key words: convex measure of risk, monetary utility function, optimal maxmin control, stochastic differential game, HJBI equation, jump diffusion market, Nash equilibrium.

Mathematics Subject Classification (2000): 93E20, 60G51, 60HXX, 60J75, 91A15, 91A23, 91B28, 62P05

\section{Introduction}

We study the problem of minimizing convex risk measures in a Lévy market. The paper [2] introduced the concept of a coherent risk measures as a measure which is sub-additive, positive homogeneous, monotonic and translation invariant. However, in [8] and [6], the idea was extended to relax the subadditivity and positive homogeneity and substitute these with the convex property. It was observed in [19] that the negative of a convex risk measure can be interpreted

\footnotetext{
${ }^{1}$ Department of Mathematics and Applied Mathematics, University of KwaZulu-Natal, Westville Campus, P.Bag X54001,Durban 4000, South Africa.

${ }^{2}$ Department of Mathematics, University of Zimbabwe, Box MP 167, Mount Pleasant, Harare, Zimbabwe. E-mail: mataramvuras@ukzn.ac.za

${ }^{3}$ Center of Mathematics for Applications (CMA), Department of Mathematics, University of Oslo, P.O. Box 1053 Blindern, N-0316 Oslo, Norway. E-mail: oksendal@math.uio.no

${ }^{4}$ Norwegian School of Economics and Business Administration, Helleveien 30, N-5045 Bergen, Norway
} 
as a special utility function termed monetary utility function. As a result, risk minimization leads to a max-min problem whose solution we are interested to find.

This problem then extends to solving a zero-sum stochastic differential game between an agent and a market. In order to simplify the max-min problem that we get in the process, we formulate and prove a general Hamilton-JacobiBellman-Isaacs (HJBI) equation for two player stochastic differential games.

Our paper is designed as follows: In the next section we describe the jump diffusion market model we want to deal with, and we formulate the risk minimizing problem as a zero-sum stochastic differential game problem (Problem 2.4). Then in Section 3 we state and prove an HJBI equation for such games (Theorem 3.2). In Section 4 we apply Theorem 3.2 to study some risk minimizing portfolio examples. Finally, in Section 5 we extend our approach to cover general stochastic differential games, not necessarily of zero-sum type, and we prove an HJBI equation for determination of the Nash equilibria of such.

\section{The market model}

Let $\eta(t)=\eta(t, \omega) ;(t, \omega) \in[0, \infty) \times \Omega$ be a Lévy process on a filtered probability space $\left(\Omega, \mathcal{F},\left\{\mathcal{F}_{t}\right\}_{t \geq 0}, P\right)$ satisfying the usual conditions. Let $N(d t, d z)$ be the jump measure of $\eta(\cdot)$ and let

$$
\nu(V)=E[N((0,1], V)] ; \quad V \subset \mathbb{R} \backslash\{0\} \text { Borel set }
$$

be the Lévy measure of $\eta(\cdot)$, where $E[\cdot]$ denotes expectation with respect to $P$. Define

$$
\tilde{N}(d t, d z)=N(d t, d z)-\nu(d z) d t
$$

the compensated Poisson random measure of $\eta(\cdot)$. We assume for simplicity that

$$
E\left[\eta^{2}(t)\right]<\infty \text { for all } t \geq 0 .
$$

Then by the Lévy-Itô decomposition theorem $\eta(\cdot)$ has the form

$$
\eta(t)=a t+b B(t)+\int_{0}^{t} \int_{\mathbb{R}} z \tilde{N}(d s, d z) ; \quad t \geq 0
$$

where $a, b$ are constants and $B(t)$ is a standard Brownian motion. In view of this we see that when we deal with models involving integration with respect to $\eta(t)$ it is natural to consider linear combinations of $d t$-integrals, $d B(t)$-integrals (i.e. classical Itô integrals) and $\tilde{N}(d t, d z)$-integrals. This leads to Lévy-Itô processes $X(t)$ of the form

$$
d X(t)=\alpha(t, \omega) d t+\beta(t, \omega) d B(t)+\int_{\mathbb{R}} \gamma(t, z) \tilde{N}(d t, d z)
$$


where $\alpha, \beta$ and $\gamma$ are $\mathcal{F}_{t}$-predictable processes satisfying

$$
\int_{0}^{T}\left\{|\alpha(t)|+\beta^{2}(t)+\int_{\mathbb{R}} \gamma^{2}(t, z) \nu(d z)\right\} d t<\infty \quad \text { for all } T<\infty .
$$

We refer to [1] for more information about the stochastic calculus of Lévy processes. See also [25, Chapter 1].

We now consider such a model in finance: Let $\alpha, \beta$ and $\gamma$ be as above, fix $T>0$ and let $r(t)=r(t, \omega)$ be adapted with $\int_{0}^{T}|r(t)| d t<\infty$ a.s. Suppose there are two investment possibilities:

(i) a risk free asset (e.g. a bond), with unit price $S_{0}(t)$ at time $t$ given by

$$
d S_{0}(t)=r(t) S_{0}(t) d t ; \quad S_{0}(0)=1
$$

(ii) a risky asset (e.g. a stock), with unit price $S_{1}(t)$ given by

$$
d S_{1}(t)=S_{1}\left(t^{-}\right)\left[\alpha(t) d t+\beta(t) d B(t)+\int_{\mathbb{R}} \gamma(t, z) \tilde{N}(d t, d z)\right] ; \quad S_{1}(0)>0
$$

where we assume that $\gamma(t, z)>-1$ for a.a. $t, z$, a.s.

Let $\pi(t)$ be a portfolio, giving the proportion of the total wealth $V(t)$ invested in the stocks at time $t$. Then the dynamics of the corresponding wealth process $V(t)=V^{(\pi)}(t)$ is

$$
\begin{aligned}
d V^{(\pi)}(t)= & V^{(\pi)}\left(t^{-}\right)[\{(1-\pi(t)) r(t)+\pi(t) \alpha(t)\} d t \\
& \left.+\pi(t) \beta(t) d B(t)+\pi\left(t^{-}\right) \int_{\mathbb{R}} \gamma(t, z) \tilde{N}(d t, d z)\right] ; \quad V^{(\pi)}(0)>0
\end{aligned}
$$

We require that $\pi\left(t^{-}\right) \gamma(t, z)>-1$ for a.a. $t, z$, a.s. and that

$$
\int_{0}^{T}\left\{|(1-\pi(t)) r(t)|+|\pi(t) \alpha(t)|+\pi^{2}(t) \beta^{2}(t)+\pi^{2}(t) \int_{\mathbb{R}} \gamma^{2}(t, z) \nu(d z)\right\} d t<\infty \text { a.s. }
$$

We now pose the following problem:

(2.6) Find the portfolio $\pi(t)$ that minimizes the risk of the terminal wealth $V^{(\pi)}(T)$.

As a measure of risk we will use the convex risk measure, introduced in [8] and [6]. It is a generalization of the concept of a coherent risk measure, introduced in [2]. 
Definition $2.1([8],[6])$ Let $\mathbb{F}$ be the family of all lower bounded $\mathcal{F}_{T}$-measurable random variables. A convex risk measure on $\mathbb{F}$ is a map $\rho: \mathbb{F} \rightarrow \mathbb{R}$ such that

(i) (convexity) $\rho(\lambda X+(1-\lambda) Y) \leq \lambda \rho(X)+(1-\lambda) \rho(Y)$ for all $X, Y \in \mathbb{F}$, $\lambda \in(0,1)$,

(ii) (monotonicity) If $X \leq Y, X, Y \in \mathbb{F}$, then $\rho(X) \geq \rho(Y)$,

(iii) (translation invariance) If $X \in \mathbb{F}$ and $m \in \mathbb{R}$ then

$$
\rho(X+m)=\rho(X)-m .
$$

In view of the general representation formula for convex risk measures (see e.g. [8, Theorem 9]), we will assume that the risk measure $\rho$ that we consider, is of the following type:

$$
\rho(X)=\sup _{Q \in \mathcal{M}_{a}}\left\{E_{Q}[-X]-\zeta(Q)\right\}
$$

(where $E_{Q}$ denotes expectation with respect to $Q$ ), for some family $\mathcal{M}_{a}$ of measures $Q$ which are absolutely continuous with respect to $P$ and for some "penalty" function $\zeta: \mathcal{M}_{a} \rightarrow \mathbb{R}$. (See also [2], [4], [6] and [9].)

One possible choice of penalty function $\zeta(Q)$ is the relative entropy of $Q$ with respect to $P$, defined by

$$
\zeta(Q):=I(Q ; P):=E\left[\frac{d Q}{d P} \log \frac{d Q}{d P}\right] .
$$

(See e.g. [13].)

A natural choice of the family $\mathcal{M}_{a}$ is the set of all measures $Q=Q_{\theta}$ of Girsanov transformation type, i.e.

$$
d Q_{\theta}(\omega)=Z_{\theta}(T) d P(\omega) \quad \text { on } \mathcal{F}_{T}
$$

where $\theta=\left(\theta_{0}, \theta_{1}\right)$ and

$$
d Z_{\theta}(t)=Z_{\theta}\left(t^{-}\right)\left[-\theta_{0}(t) d B(t)-\int_{\mathbb{R}} \theta_{1}(t, z) \tilde{N}(d t, d z)\right] ; \quad Z_{\theta}(0)=1 .
$$

Here $\theta_{0}(t)$ and $\theta_{1}(t, z)$ are $\mathcal{F}_{t}$-adapted processes, $\theta_{1}(t, z)<1$ for a.a. $t, z$ and

$$
\int_{0}^{T}\left\{\theta_{0}^{2}(t)+\int_{\mathbb{R}} \theta_{1}^{2}(t, z) \nu(d z)\right\} d t<\infty \quad \text { a.s. }
$$

Then by the Itô formula the solution of the stochastic differential equation (2.10) 
is

$$
\begin{aligned}
Z_{\theta}(t) & =\exp \left[-\int_{0}^{t} \theta_{0}(s) d B(s)-\frac{1}{2} \int_{0}^{t} \theta_{0}^{2}(s) d s\right. \\
& +\int_{0}^{t} \int_{\mathbb{R}} \log \left(1-\theta_{1}(s, z)\right) \tilde{N}(d s, d z) \\
& +\int_{0}^{t} \int_{\mathbb{R}}\left\{\log \left(1-\theta_{1}(s, z)\right)+\theta_{1}(s, z)\right\} \nu(d z) d s ; \quad 0 \leq t \leq T .
\end{aligned}
$$

Remark 2.2 In particular, if $\theta_{0}(t), \theta_{1}(t, z)$ are such that

$$
E[Z(T)]=1
$$

and

$$
\beta(t) \theta_{0}(t)+\int_{\mathbb{R}} \gamma(t, z) \theta_{1}(t, z) \nu(d z)=\alpha(t)-r(t) \quad \text { for a.a. } t,
$$

then $d Q_{\theta}(\omega)=Z_{\theta}(T) d P(\omega)$ is an equivalent local martingale measure (see e.g. [25, Ch. 1]), but we do not assume this here.

In view of (2.7) we can now make our risk minimizing portfolio problem more precise:

(2.15) Given a convex risk measure $\rho$ of the form (2.7) find the portfolio $\pi$ which minimizes

$$
\sup _{Q \in \mathcal{M}_{a}}\left\{E_{Q}\left[-V^{(\pi)}(T)\right]-\zeta(Q)\right\}
$$

A related concept is the following (see e.g. [19]):

Definition 2.3 A monetary utility function is a map $U: \mathbb{F} \rightarrow \mathbb{R}$ with the following properties:

(i) (concavity) $U(\lambda X+(1-\lambda) Y) \geq \lambda U(X)+(1-\lambda) U(Y)$, for all $X, Y \in \mathbb{F}$, $\lambda \in(0,1)$.

(ii)' (monotonicity) If $X \leq Y, X, Y \in \mathbb{F}$, then $U(X) \leq U(Y)$.

(iii)' (translation invariance) If $X \in \mathbb{F}$ and $m \in \mathbb{R}$ then $U(X+m)=U(X)+m$.

It is easily seen that if $\rho$ is a convex risk measure, then $U(X):=-\rho(X)$ is a monetary utility function and conversely. Therefore, in view of (2.7) we have the following general representation of a monetary utility function:

$$
U(X)=\inf _{Q \in \mathcal{M}_{a}}\left\{E_{Q}[X]+\zeta(Q)\right\}
$$

Using this our risk minimizing portfolio problem (2.15) becomes equivalent to the following monetary utility maximizing problem: 
Problem 2.4 Find

$$
\Phi:=\sup _{\pi}\left(\inf _{Q \in \mathcal{M}_{a}}\left\{E_{Q}\left[V^{(\pi)}(T)\right]+\zeta(Q)\right\}\right)
$$

and find the optimal $\pi^{*}$ and $Q^{*}$ such that

$$
\Phi=E_{Q^{*}}\left[V^{\left(\pi^{*}\right)}(T)\right]+\zeta\left(Q^{*}\right) .
$$

Remark 2.5 We may regard this as a game theoretic problem: The measure $Q$ is the control of player number 1 (the "market"), while the portfolio $\pi(t)$ is the control of player number 2 (the representative agent/trader).

Problem 2.4 is a special case of a stochastic zero-sum differential game. In the next sections we present a general formulation of such a game and we give a general solution method in terms of a Hamilton-Jacobi-Bellman-Isaacs (HJBI) equation. See Theorem 3.2 (for the zero-sum case) and Theorem 5.2 (for the Nash equilibrium case), which together with Example 4.1 and Theorem 5.4 are our main new results. To the best of our knowledge this is the first time differential games of this type are studied in a jump diffusion context. For deterministic systems similar differential games have been studied by many authors. See e.g. [16], [5], [21] and [7] and the references therein. See also [11], [14], [15], [22], [28] and [31] for papers of related interest.

A nice presentation of games in a general Markov process context is given in [18]. For information on the existence of a value function in zero-sum stochastic differential games see [10].

Problem 2.4 is related to what is also known as a robust utility maximization problem. See e.g. [12] or [30] for a duality approach to such problems. A stochastic control approach (different from ours) is presented in [3]. Another paper of related interest is [20], where certain worst case crash scenarios are studied using a stochstic control approach.

Risk measures can also be represented by g-expectations and hence by solutions of backward stochastic differential equations. For more information we refer to [26] and the references therein.

\section{An HJBI equation for zero-sum stochastic dif- ferential games}

Suppose the equation for the state $Y(t)=Y^{(u)}(t)$ at time $t$ has the form

$$
\begin{aligned}
d Y(t)= & b\left(Y(t), u_{0}(t)\right) d t+\sigma\left(Y(t), u_{0}(t)\right) d B(t) \\
& +\int_{\mathbb{R}^{k}} \gamma\left(Y\left(t^{-}\right), u_{1}\left(t^{-}, z\right), z\right) \tilde{N}(d t, d z) ; \quad Y(0)=y \in \mathbb{R}^{k},
\end{aligned}
$$

Here $b: \mathbb{R}^{k} \times K \rightarrow \mathbb{R}^{k}, \sigma: \mathbb{R}^{k} \times K \rightarrow \mathbb{R}^{k \times k}$ and $\gamma: \mathbb{R}^{k} \times K \times \mathbb{R}^{k} \rightarrow$ $\mathbb{R}^{k \times k}$ are given functions, $B(t)$ is a $k$-dimensional Brownian motion, $\tilde{N}(\cdot, \cdot)=$ 
$\left(\tilde{N}_{1}(\cdot, \cdot), \ldots, \tilde{N}_{k}(\cdot, \cdot)\right)$ are $k$-independent compensated Poisson random measures and $K$ is a given subset of $\mathbb{R}^{p}$.

The process $u_{0}(t)=u_{0}(t, \omega)$ and $u_{1}(t, z)=u_{1}(t, z, \omega)$ are our control processes, assumed to be cadlag and adapted to the filtration $\mathcal{F}_{t}$ generated by the driving processes $B(\cdot)$ and $\tilde{N}(\cdot, \cdot)$ (as usual augmented with all the $P$-null sets), and with values $u_{0}(t) \in K, u_{1}(t, z) \in K$ for a.a. $t, z$, a.s. We put $u=\left(u_{0}, u_{1}\right)$ and we call $Y^{(u)}(\cdot)$ a controlled jump diffusion. We refer to [25] for more information about stochastic control of jump diffusions.

Let $f: \mathbb{R}^{k} \times K \rightarrow \mathbb{R}$ and $g: \mathbb{R}^{k} \rightarrow \mathbb{R}$ be given functions, called the profit rate and bequest function, respectively. We assume that we are given a family $\mathcal{A}$ of admissible controls, contained in the set of controls $u(\cdot)$ such that (3.1) has a unique strong solution and such that

$$
E^{y}\left[\int_{0}^{\tau_{\mathcal{S}}}\left|f\left(Y(t), u_{0}(t)\right)\right| d t+\left|g\left(Y\left(\tau_{\mathcal{S}}\right)\right)\right|\right]<\infty
$$

for all $y \in \mathcal{S}$, where $\mathcal{S} \subset \mathbb{R}^{k}$ is a given open set (called the solvency region),

$$
\tau_{\mathcal{S}}=\inf \{t>0 ; Y(t) \notin \mathcal{S}\}
$$

is the bankruptcy time and $E^{y}$ denotes expectation given that $Y(0)=y \in \mathbb{R}^{k}$.

For $u \in \mathcal{A}$ we define the performance functional $J^{u}(y)$ by

$$
J^{u}(y)=E^{y}\left[\int_{0}^{\tau_{\mathcal{S}}} f\left(Y(t), u_{0}(t)\right) d t+g\left(Y\left(\tau_{\mathcal{S}}\right)\right)\right] .
$$

Here, and in (3.2), we interpret $g\left(Y\left(\tau_{\mathcal{S}}\right)\right)$ as 0 if $\tau_{\mathcal{S}}=\infty$.

Now suppose that the controls $u_{0}(t)$ and $u_{1}(t, z)$ have the form

$$
\begin{aligned}
& u_{0}(t)=\left(\theta_{0}(t), \pi_{0}(t)\right) ; \quad t \geq 0 \\
& u_{1}(t, z)=\left(\theta_{1}(t, z), \pi_{1}(t, z)\right) ; \quad(t, z) \in[0, \infty) \times \mathbb{R}^{k}
\end{aligned}
$$

Just as in the example discussed in Section 2 we may regard $\theta:=\left(\theta_{0}, \theta_{1}\right)$ as the control of player number 1 , while $\pi:=\left(\pi_{0}, \pi_{1}\right)$ is the control of player number 2.

Let $\Theta$ and $\Pi$ be given families of admissible controls $\theta=\left(\theta_{0}, \theta_{1}\right)$ and $\pi=$ $\left(\pi_{0}, \pi_{1}\right)$, respectively. Let $K_{1}, K_{2}$ be two sets such that $\theta(t, z) \in K_{1}$ and $\pi(t, z) \in K_{2}$ for a.a. $t, z, \omega$. The general zero-sum stochastic differential game problem is the following

Problem 3.1 Find $\Phi(y)$ and $\left(\theta^{*}, \pi^{*}\right) \in \Theta \times \Pi$ such that

$$
\Phi(y)=\sup _{\pi \in \Pi}\left(\inf _{\theta \in \Theta} J^{\theta, \pi}(y)\right)=J^{\left(\theta^{*}, \pi^{*}\right)}(y) .
$$

The function $\Phi$ is called the value function and $\left(\theta^{*}, \pi^{*}\right)$ is called an optimal control (if it exists). 
In classical stochastic control theory it is well-known that under mild conditions Markov controls can give just as good performance as the more general adapted controls. See e.g. [23, Theorem 11.2.3]. In view of this we will restrict ourselves to consider only Markov controls in Problem 3.1. Hence we assume that

$$
\begin{aligned}
& \theta_{0}(t)=\bar{\theta}_{0}(Y(t)), \quad \pi_{0}(t)=\bar{\pi}_{0}(Y(t)), \quad \theta_{1}(t, z)=\bar{\theta}_{1}(Y(t), z) \\
& \text { and } \pi_{1}(t, z)=\bar{\pi}_{1}(Y(t), z)
\end{aligned}
$$

for some function $\bar{\theta}_{0}: \mathcal{S} \rightarrow K_{1}, \bar{\pi}_{0}: \mathcal{S} \rightarrow K_{2}, \bar{\theta}_{1}: \mathcal{S} \times \mathbb{R}^{k} \rightarrow K_{1}$ and $\bar{\pi}_{1}$ : $\mathcal{S} \times \mathbb{R}^{k} \rightarrow K_{2}$.

As customary we do not distinguish notationally between $\theta_{0}$ and $\bar{\theta}_{0}, \pi_{0}$ and $\bar{\pi}_{0}, \theta_{1}$ and $\bar{\theta}_{1}$ and $\pi_{1}$ and $\bar{\pi}_{1}$. Thus our controls can simply be identified with (deterministic) functions $\theta_{0}(y), \pi_{0}(y), \theta_{1}(y, z)$ and $\pi_{1}(y, z) ; y \in \mathcal{S}, z \in \mathbb{R}^{k}$ (sometimes also called feedback controls).

When the control $u=(\theta, \pi) \in \Theta \times \Pi$ is Markovian, the corresponding system (3.1) becomes a jump diffusion, whose generator $A^{\theta, \pi}$ is given by

$$
\begin{aligned}
A^{\theta, \pi} \varphi(y)=\sum_{i=1}^{k} b_{i}\left(y, \theta_{0}(y), \pi_{0}(y)\right) \frac{\partial \varphi}{\partial y_{i}}(y) \\
\quad+\frac{1}{2} \sum_{i, j=1}^{k}\left(\sigma \sigma^{T}\right)_{i j}\left(y, \theta_{0}(y), \pi_{0}(y)\right) \frac{\partial^{2} \varphi}{\partial y_{i} \partial y_{j}}(y) \\
\quad+\sum_{j=1}^{k} \int_{\mathbb{R}}\left\{\varphi\left(y+\gamma^{(j)}\left(y, \theta_{1},\left(y, z_{j}\right), \pi_{1}\left(y, z_{j}\right), z_{j}\right)\right)-\varphi(y)\right. \\
\left.\quad-\nabla \varphi(y) \cdot \gamma^{(j)}\left(y, \theta_{1}\left(y, z_{j}\right), \pi_{1}\left(y, z_{j}\right), z_{j}\right)\right\} \nu_{j}\left(d z_{j}\right) ;
\end{aligned}
$$

where $\varphi \in C_{0}^{2}\left(\mathbb{R}^{k}\right)$ (the twice continuously differentiable functions with compact support on $\left.\mathbb{R}^{k}\right)$. Here $\nabla \varphi=\left(\frac{\partial \varphi}{\partial y_{1}}, \ldots, \frac{\partial \varphi}{\partial y_{k}}\right)$ is the gradient of $\varphi$ and $\gamma^{(j)}$ is the $j$ 'th column of the $k \times k$ matrix $\gamma$.

We let $\mathcal{T}$ denote the set of all $\mathcal{F}_{t}$-stopping times $\tau \leq \tau_{\mathcal{S}}$.

We can now state the first main result of this paper:

Theorem 3.2 (An HJBI equation for zero-sum differential games for jump diffusions) Suppose there exists a function $\varphi \in C^{2}(\mathcal{S}) \cap C(\overline{\mathcal{S}})$ and a Markov control $(\hat{\theta}(y), \hat{\pi}(y)) \in \Theta \times \Pi$ such that

(i) $\quad A^{\theta, \hat{\pi}(y)} \varphi(y)+f(y, \theta, \hat{\pi}(y)) \geq 0 \quad$ for all $\theta \in K_{1}, y \in \mathcal{S}$

(ii) $\quad A^{\hat{\theta}(y), \pi} \varphi(y)+f(y, \hat{\theta}(y), \pi) \leq 0 \quad$ for all $\pi \in K_{2}, y \in \mathcal{S}$

(iii) $\quad A^{\hat{\theta}(y), \hat{\pi}(y)} \varphi(y)+f(y, \hat{\theta}(y), \hat{\pi}(y))=0 \quad$ for all $y \in \mathcal{S}$

(iv) $Y^{\theta, \pi}\left(\tau_{\mathcal{S}}\right) \in \partial \mathcal{S}$ a.s. on $\left\{\tau_{\mathcal{S}}<\infty\right\}$ and $\lim _{t \rightarrow \tau_{\mathcal{S}}^{-}} \varphi\left(Y^{\theta, \pi}(t)\right)=g\left(Y^{\theta, \pi}\left(\tau_{\mathcal{S}}\right)\right) \chi_{\left\{\tau_{\mathcal{S}}<\infty\right\}}$ a.s. for all $(\theta, \pi) \in \Theta \times \Pi, y \in \mathcal{S}$

(v) the family $\left\{\varphi\left(Y^{\theta, \pi}(\tau)\right)\right\}_{\tau \in \mathcal{T}}$ is uniformly integrable, for all $y \in \mathcal{S},(\theta, \pi) \in \Theta \times \Pi$. 
Then

$$
\begin{aligned}
\varphi(y) & =\Phi(y)=\sup _{\pi \in \Pi}\left(\inf _{\theta \in \Theta} J^{\theta, \pi}(y)\right)=\inf _{\theta \in \Theta}\left(\sup _{\pi \in \Pi} J^{\theta, \pi}(y)\right) \\
& =\sup _{\pi \in \Pi} J^{\hat{\theta}, \pi}(y)=\inf _{\theta \in \Theta} J^{\theta, \hat{\pi}}(y)=J^{\hat{\theta}, \hat{\pi}}(y) ; \quad y \in \mathcal{S}
\end{aligned}
$$

and

$$
(\hat{\theta}(y), \hat{\pi}(y)) \quad \text { is an optimal (Markov) control. }
$$

Proof. Choose $(\theta, \pi) \in \Theta \times \Pi$. Then by the Dynkin formula for jump diffusions (see e.g. [25, Theorem 1.24]) we have

$$
E^{y}\left[\varphi\left(Y\left(\tau_{\mathcal{S}}^{(N)}\right)\right)\right]=\varphi(y)+E^{y}\left[\int_{0}^{\tau_{\mathcal{S}}^{(N)}} A^{\theta, \pi} \varphi(Y(t)) d t\right]
$$

where $Y(t)=Y^{\theta, \pi}(t)$ and

$$
\tau_{\mathcal{S}}^{(N)}=\tau_{\mathcal{S}} \wedge N \wedge \inf \{t>0 ;|Y(t)| \geq N\}, \quad N=1,2, \ldots
$$

(I) If we apply (3.12) to $\theta, \hat{\pi}$ and use (i) for all $y=Y(t)$ we get

$$
E^{y}\left[\varphi\left(Y\left(\tau_{\mathcal{S}}^{(N)}\right)\right)\right] \geq \varphi(y)-E^{y}\left[\int_{0}^{\tau_{\mathcal{S}}^{(N)}} f(Y(t), \theta(Y(t)), \hat{\pi}(Y(t)) d t]\right.
$$

or

$$
\varphi(y) \leq E^{y}\left[\int_{0}^{\tau_{\mathcal{S}}^{(N)}} f(Y(t), \theta(Y(t)), \hat{\pi}(Y(t))) d t+\varphi\left(Y\left(\tau_{\mathcal{S}}^{(N)}\right)\right)\right] .
$$

Letting $N \rightarrow \infty$ and using (iv) and (v) we obtain

$$
\varphi(y) \leq J^{\theta, \hat{\pi}}(y)
$$

Since this holds for all $\theta \in \Theta$ we deduce that

$$
\varphi(y) \leq \inf _{\theta \in \Theta} J^{\theta, \hat{\pi}}(y) .
$$

Hence

$$
\varphi(y) \leq \sup _{\pi \in \Pi}\left(\inf _{\theta \in \Theta} J^{\theta, \pi}(y)\right)=\Phi(y)
$$

(II) Next, if we apply (3.12) to $\hat{\theta}, \pi$, with $\pi \in \Pi$, and use (ii) for all $y=Y(t)$ we get

$$
E^{y}\left[\varphi\left(Y\left(\tau_{\mathcal{S}}^{(N)}\right)\right)\right] \leq \varphi(y)-E^{y}\left[\int_{0}^{\tau_{\mathcal{S}}^{(N)}} f(Y(t), \hat{\theta}(Y(t)), \pi(Y(t))) d t\right]
$$


or

$$
\varphi(y) \geq E^{y}\left[\int_{0}^{\tau_{\mathcal{S}}^{(N)}} f(Y(t), \hat{\theta}(Y(t)), \pi(Y(t))) d t+\varphi\left(Y\left(\tau_{\mathcal{S}}^{(N)}\right)\right)\right] .
$$

Letting $N \rightarrow \infty$ and using (iv) and (v) we obtain

$$
\varphi(y) \geq J^{\hat{\theta}, \pi}(y) \geq \inf _{\theta \in \Theta} J^{\theta, \pi}(y)
$$

Since this holds for all $\pi \in \Pi$ we deduce that

$$
\varphi(y) \geq \sup _{\pi \in \Pi}\left(\inf _{\theta \in \Theta} J^{\theta, \pi}(y)\right)=\Phi(y) .
$$

(III) Finally, we apply (3.12) to $\hat{\theta}, \hat{\pi}$ and proceed as above. Then we end up with

$$
\varphi(y)=J^{\hat{\theta}, \hat{\pi}}(y)
$$

Combining (3.17), (3.18) and (3.15) we conclude that

$$
\Phi(y) \leq \varphi(y)=J^{\hat{\theta}, \hat{\pi}}(y) \leq \Phi(y)
$$

Combining (3.16) and (3.13) we get

$$
\begin{aligned}
& \inf _{\theta \in \Theta}\left(\sup _{\pi \in \Pi} J^{\theta, \pi}(y)\right) \leq \sup _{\pi \in \Pi} J^{\hat{\theta}, \pi}(y) \leq \varphi(y) \leq \inf _{\theta \in \Theta} J^{\theta, \hat{\pi}}(y) \\
& \quad \leq \sup _{\pi \in \Pi}\left(\inf _{\theta \in \Theta} J^{\theta, \pi}(y)\right)=\Phi(y) .
\end{aligned}
$$

On the other hand, we always have

$$
\sup _{\pi \in \Pi}\left(\inf _{\theta \in \Theta} J^{\theta, \pi}(y)\right) \leq \inf _{\theta \in \Theta}\left(\sup _{\pi \in \Pi} J^{\theta, \pi}(y)\right) .
$$

Combining (3.19) and (3.20) with (3.18) we get (3.10) and (3.11).

Remark 3.3 In the case when $d Y(t)=(d t, X(t)), Y(0)=y=(s, x) \in[0, T] \times$ $\mathbb{R}^{n}$ it suffices to assume that $\varphi \in C^{1,2}\left((0, T) \times \mathbb{R}^{n}\right) \cap C(\overline{\mathcal{S}})$. This applies to Examples 4.1, 4.2 and 5.3.

Remark 3.4 In this paper we do not discuss the (important) question to what extent the value function $\Phi$ is indeed a $C^{2}$ function (or $C^{1,2}$ function) under some general conditions. However, we verify that this is the case in some specific examples (Example 4.1 and Example 5.3). It is known that under some conditions the value function $\Phi$ is a viscosity solution of the corresponding HJBI equation. See [17]. 


\section{Examples}

Example 4.1 Let us return to the market (2.3), (2.4) in Section 2. However, as suggested by [27], let us assume that the mean rate of return of the stock, $\alpha($.$) , is not given a priori, but is the consequence of the portfolio choice \pi(.) \in \Pi$ of a "representative" trader. The trader tries to maximize the expected utility of her terminal wealth by choosing her portfolio optimally, while the "market" tries to minimize this maximum expected utility by choosing $\alpha(t)$ accordingly. This leads to the min-max problem

$$
\inf _{\alpha \in \mathfrak{A}}\left(\sup _{\pi \in \Pi} E\left[U_{0}\left(V^{(\pi)}(T)\right)\right]\right)
$$

where $U_{0} \in C^{2}(0, \infty)$ is a given utility function (in the usual sense, not necessarily monetary) and $\mathfrak{A}, \Pi$ are given families of admissible processes $\alpha(t), \pi(t)$. For simplicity we assume that $\gamma(t, z)=z>-1$ a.s. $\nu$ and that $\beta$ is constant. We assume that if $(\alpha, \pi) \in \mathfrak{A} \times \Pi$ then

$$
E\left[\int_{0}^{T}\left\{|(1-\pi(t)) r(t)|+|\pi(t) \alpha(t)|+\pi^{2}(t) \int_{\mathbb{R}} z^{2} \nu(d z)\right\} d t\right]<\infty,
$$

which guarantees that $V^{(\pi)}(t)>0$ for all $t$.

To put this problem into the framework of Section 3 we define the process $Y(t)=\left(Y_{0}(t), Y_{1}(t)\right)$ by

$$
d Y_{0}(t)=d t ; \quad Y_{0}(0)=y_{0}=s \in \mathbb{R}
$$

and (see $(2.5))$

$$
\begin{aligned}
& d Y_{1}(t)=d V^{(\pi)}(t)=Y_{1}\left(t^{-}\right)[\{(1-\pi(t)) r(t)+\pi(t) \alpha(t)\} d t \\
& \left.+\pi(t) \beta d B(t)+\pi\left(t^{-}\right) \int_{\mathbb{R}} z \tilde{N}(d t, d z)\right] ; \quad Y_{1}(0)=y_{1}=x>0 .
\end{aligned}
$$

Then problem (4.1) can be formulated as follows:

Find $\left(\alpha^{*}, \pi^{*}\right) \in \mathfrak{A} \times \Pi$ and $\Phi(y)=\Phi(s, x)$ such that

$$
\Phi(s, x)=\inf _{\alpha \in \mathfrak{A}}\left(\sup _{\pi \in \Pi} E^{s, x}\left[U_{0}\left(Y_{1}^{(\alpha, \pi)}(T)\right)\right]\right)=E^{s, x}\left[U_{0}\left(Y_{1}^{\left(\alpha^{*}, \pi^{*}\right)}(T)\right)\right] .
$$

In this case the generator $A^{\alpha, \pi}$ has the form

$$
\begin{array}{r}
A^{\alpha, \pi} \varphi(s, x)=\frac{\partial \varphi}{\partial s}+x[(1-\pi) r(s)+\pi \alpha] \frac{\partial \varphi}{\partial x}+\frac{1}{2} \beta^{2} \pi^{2} x^{2} \frac{\partial^{2} \varphi}{\partial x^{2}} \\
\quad+\int_{\mathbb{R}}\left\{\varphi(s, x+\pi x z)-\varphi(s, x)-\frac{\partial \varphi}{\partial x}(s, x) \cdot \pi x z\right\} \nu(d z)
\end{array}
$$


and the HJBI equation for this problem can be written (in compact form)

$$
\left\{\begin{array}{l}
\inf _{\alpha \in K_{1}}\left(\sup _{\pi \in K_{2}}\left\{A^{\alpha, \pi} \varphi(s, x)\right\}\right)=0 ; \quad s<T \\
\varphi(T, x)=U_{0}(x)
\end{array}\right.
$$

Note that we only need to have $\varphi \in C^{1,2}((0, T) \times(0, \infty))$ in this case. See Remark 3.3.

Let us try the following function:

$$
\varphi(s, x)=U_{0}\left(x \exp \left(\int_{s}^{T} r(t) d t\right)\right) .
$$

This gives

$$
\begin{aligned}
& A^{\alpha, \pi} \varphi(s, x)=-U_{0}^{\prime}\left(x \exp \left(\int_{s}^{T} r(t) d t\right)\right) x \exp \left(\int_{s}^{T} r(t) d t\right) r(s) \\
& +x[(1-\pi) r(s)+\pi \alpha] U_{0}^{\prime}\left(x \exp \left(\int_{s}^{T} r(t) d t\right)\right) \exp \left(\int_{s}^{T} r(t) d t\right) \\
& +\frac{1}{2} \beta^{2} \pi^{2} x^{2} U_{0}^{\prime \prime}\left(x \exp \left(\int_{s}^{T} r(t) d t\right)\right) \exp \left(2 \int_{s}^{T} r(t) d t\right) \\
& +\int_{\mathbb{R}}\left\{U_{0}\left((x+\pi x z) \exp \left(\int_{s}^{T} r(t) d t\right)\right)-U_{0}\left(x \exp \left(\int_{s}^{T} r(t) d t\right)\right)\right. \\
& \left.-U_{0}^{\prime}\left(x \exp \left(\int_{s}^{T} r(t) d t\right)\right) \exp \left(\int_{s}^{T} r(t) d t\right) \pi x z\right\} \nu(d z)
\end{aligned}
$$

Maximizing over $\pi$ we get the following first order condition for the maximum point $\hat{\pi}=\hat{\pi}(\alpha)$ :

(4.9) $(\alpha-r(s)) U_{0}^{\prime}\left(x \exp \left(\int_{s}^{T} r(t) d t\right)\right)$

$$
\begin{aligned}
& +\beta^{2} \hat{\pi} x U_{0}^{\prime \prime}\left(x \exp \left(\int_{s}^{T} r(t) d t\right)\right) \exp \left(\int_{s}^{T} r(t) d t\right) \\
& +\int_{\mathbb{R}}\left\{U_{0}^{\prime}\left((x+\hat{\pi} x z) \exp \left(\int_{s}^{T} r(t) d t\right)\right)-U_{0}^{\prime}\left(x \exp \left(\int_{s}^{T} r(t) d t\right)\right)\right\} z \nu(d z)=0
\end{aligned}
$$

Substituting $\pi=\hat{\pi}(\alpha)$ into (4.7) and minimizing over $\alpha$ gives the first order 
condition for the minimum point $\alpha=\hat{\alpha}$ :

$$
\begin{aligned}
& {\left[-r(s) \hat{\pi}^{\prime}(\alpha)+\hat{\pi}(\alpha)+\alpha \hat{\pi}^{\prime}(\alpha)\right] U_{0}^{\prime}\left(x \exp \left(\int_{s}^{T} r(t) d t\right)\right)} \\
& +\beta^{2} \hat{\pi}(\alpha) \hat{\pi}^{\prime}(\alpha) x U_{0}^{\prime \prime}\left(x \exp \left(\int_{s}^{T} r(t) d t\right)\right) \exp \left(\int_{s}^{T} r(t) d t\right) \\
& +\int_{\mathbb{R}}\left\{U_{0}^{\prime}\left((x+\hat{\pi}(\alpha) x z) \exp \left(\int_{s}^{T} r(t) d t\right)\right)\right. \\
& \left.-U_{0}^{\prime}\left(x \exp \left(\int_{s}^{T} r(t) d t\right)\right)\right\} \hat{\pi}(\alpha) z \nu(d z)=0
\end{aligned}
$$

Combining (4.9) with (4.8) we get

$$
\hat{\pi}(\hat{\alpha})=0 .
$$

Substituting $\alpha=\hat{\alpha}$ and $\hat{\pi}(\hat{\alpha})=0$ in (4.8) we obtain

$$
\hat{\alpha}(s)=r(s) .
$$

With these values of $\hat{\alpha}, \hat{\pi}(\hat{\alpha})$, we easily verify that

$$
A^{\hat{\alpha}, \hat{\pi}(\hat{\alpha})} \varphi(s, x)=0
$$

which proves that the value function is indeed

$$
\Phi(s, x)=\varphi(s, x)=U_{0}\left(x \exp \left(\int_{s}^{T} r(t) d t\right)\right)
$$

We conclude that in this virtual game between the trader and market, the "market" reacts to the trader's optimal portfolio choice by choosing

$$
\alpha=\hat{\alpha}(t)=r(t) \quad \text { for all } t \in[0, T] .
$$

This corresponds to changing the original probability measure $P$ to some equivalent martingale measure $Q$, which is in agreement with the principle of arbitrage free option pricing. See [27] for more discussion on this. The result above is an extension of the result in [27] to (Markovian) stochastic processes $r(s), \alpha(s), \beta(s)$ and to the jump diffusion case.

Example 4.2 We now use Theorem 3.2 to study Problem 2.4. First, let us assume for simplicity that in the market (2.3)-(2.4) we have

$$
r(t)=0, \quad \alpha(t)=\alpha(\text { constant }), \quad \beta(t)=\beta \text { (constant), } \quad \gamma(t, z)=0
$$

i.e. the market is a classical Black-Scholes market.

Let $\mathcal{M}_{a}$ consist of all probability measure $Q=Q_{\theta}$ of the form 


$$
d Q_{\theta}=\exp \left(-\int_{0}^{T} \theta_{0}(s) d B(s)-\frac{1}{2} \int_{0}^{T} \theta_{0}^{2}(s) d s\right) d P(\omega) \quad \text { on } \mathcal{F}_{T}
$$

where $\theta_{0}(s)$ is $\mathcal{F}_{s}$-adapted, $E\left[\exp \left(\frac{1}{2} \int_{0}^{T} \theta_{0}^{2}(s) d s\right)\right]<\infty$.

We put Problem 2.4 into the framework of Section 3 by defining

$$
d Y(t)=\left(d Y_{0}(t), d Y_{1}(t), d Y_{2}(t)\right) ; \quad Y(0)=y=\left(s, y_{1}, y_{2}\right),
$$

where

$$
\begin{array}{ll}
d Y_{0}(t)=d t ; \quad Y_{0}(0)=s \in \mathbb{R} & \\
d Y_{1}(t)=d V^{(\pi)}(t)=Y_{1}(t)[\alpha \pi(t) d t+\beta \pi(t) d B(t)] ; & Y_{1}(0)=y_{1}>0 \\
d Y_{2}(t)=-\theta(t) Y_{2}(t) d B(t) ; \quad Y_{2}(0)=y_{2}>0 &
\end{array}
$$

and where we have put $\theta_{0}=\theta, \pi_{0}=\pi$, for simplicity of notation.

Assume that the penalty function has the form

$$
\zeta\left(Q_{\theta}\right)=E\left[\zeta_{0}\left(\frac{d Q_{\theta}}{d P}\right)\right]=E\left[\zeta_{0}\left(Y_{2}(T)\right)\right] \text { for some function } \zeta_{0}: \mathbb{R} \rightarrow \mathbb{R} .
$$

Then Problem 2.4 gets the form

$$
\Phi(y)=\Phi\left(s, y_{1}, y_{2}\right)=\sup _{\pi \in \Pi}\left(\inf _{\theta \in \Theta} E^{s, y_{1}, y_{2}}\left[Y_{1}(T) Y_{2}(T)+\zeta_{0}\left(Y_{2}(T)\right)\right]\right) .
$$

In this case the generator of $Y(\cdot)$ becomes

$$
\begin{aligned}
& A^{\theta, \pi} \varphi(y)=\frac{\partial \varphi}{\partial s}+y_{1} \alpha \pi \frac{\partial \varphi}{\partial y_{1}}+\frac{1}{2} y_{1}^{2} \beta^{2} \pi^{2} \frac{\partial^{2} \varphi}{\partial y_{1}^{2}} \\
& \quad-y_{1} y_{2} \beta \theta \pi \frac{\partial^{2} \varphi}{\partial y_{1} \partial y_{2}}+\frac{1}{2} y_{2}^{2} \theta^{2} \frac{\partial^{2} \varphi}{\partial y_{2}^{2}} ; \quad \varphi \in C_{0}^{2}\left(\mathbb{R}^{3}\right) .
\end{aligned}
$$

Then the corresponding HJBI equation becomes

$$
\left\{\begin{array}{l}
\sup _{\pi \in K_{2}}\left(\inf _{\theta \in K_{1}} A^{\theta, \pi} \varphi\left(s, y_{1}, y_{2}\right)\right)=0 ; \quad s<T \\
\varphi\left(T, y_{1}, y_{2}\right)=y_{1} y_{2}+\zeta_{0}\left(y_{2}\right)
\end{array}\right.
$$

To study this equation we first fix $\pi \in K_{2}$ and minimize

$$
h(\theta):=-y_{1} y_{2} \beta \theta \pi \varphi_{12}+\frac{1}{2} y_{2}^{2} \theta^{2} \varphi_{22}
$$

with respect to $\theta$. (Here $\varphi_{12}=\frac{\partial^{2} \varphi}{\partial y_{1} \partial y_{2}}(y)$ etc.) The minimum is attained at

$$
\theta=\hat{\theta}(y)=\frac{y_{1} \beta \pi \varphi_{12}}{y_{2} \varphi_{22}} \quad\left(\text { if } \varphi_{22}>0\right) .
$$


Substituting this into (4.18) we get to maximize

$$
k(\pi):=y_{1} \alpha \pi \varphi_{1}+\frac{1}{2} y_{1}^{2} \beta^{2} \pi^{2}\left(\varphi_{11}-\frac{\varphi_{12}^{2}}{\varphi_{22}}\right)
$$

with respect to $\pi$. If $\varphi_{11}<\frac{\varphi_{12}^{2}}{\varphi_{22}}$ the maximum is attained when

$$
\pi=\hat{\pi}(y)=\frac{\alpha \varphi_{1} \varphi_{22}}{y_{1} \beta^{2}\left(\varphi_{12}^{2}-\varphi_{11} \varphi_{22}\right)} .
$$

Substituting (4.19) and (4.20) into (4.18) we get the following non-linear partial differential equation for the unknown value function $\varphi$ :

$$
\left\{\begin{array}{l}
\frac{\partial \varphi}{\partial s}+\frac{\alpha^{2} \varphi_{1}^{2} \varphi_{22}}{2 \beta^{2}\left(\varphi_{12}^{2}-\varphi_{11} \varphi_{22}\right)}=0 ; \quad s<T \\
\varphi\left(T, y_{1}, y_{2}\right)=y_{1} y_{2}+\zeta_{0}\left(y_{2}\right)
\end{array}\right.
$$

If the solution $\varphi$ of this equation exists and the corresponding $\hat{\theta}(y), \hat{\pi}(y)$ given by (4.19)-(4.20) satisfies all the requirements of Theorem 3.2, then $\varphi=\Phi$ and $\left(\theta^{*}, \pi^{*}\right):=(\hat{\theta}, \hat{\pi})$ is an optimal (Markov) control.

Example 4.3 Let us again use Theorem 3.2 to study Problem 2.4, but in this case assuming that in the market (2.3)-(2.4) we have

$$
r(t)=0, \quad \alpha(t)=\alpha \text { (constant), } \quad \beta=0 \quad \text { and } \quad \gamma(t, z)=z .
$$

Now let $\mathcal{M}_{a}$ consist of all probability measures $Q=Q_{\theta}$ of the form

$$
d Q_{\theta}(\omega)=Z_{\theta_{1}}(T) d P(\omega) \quad \text { on } \mathcal{F}_{T},
$$

where

$$
\begin{aligned}
Z_{\theta_{1}}(t) & =\exp \left[\int_{0}^{t} \int_{\mathbb{R}} \log \left(1-\theta_{1}(s, z)\right) \tilde{N}(d s, d z)\right. \\
& +\int_{0}^{t} \int_{\mathbb{R}}\left\{\log \left(1-\theta_{1}(s, z)\right)+\theta_{1}(s, z)\right\} \nu(d z) d s ; \quad 0 \leq t \leq T .
\end{aligned}
$$

Then, by the Itô formula,

$$
d Z_{\theta_{1}}(t)=-Z_{\theta_{1}}\left(t^{-}\right) \int_{\mathbb{R}} \theta_{1}(t, z) \tilde{N}(d t, d z) .
$$

Therefore, to put Problem 2.4 into the framework of Section 3 we define

$$
Y(t)=\left(Y_{0}(t), Y_{1}(t), Y_{2}(t)\right), \quad Y(0)=\left(s, y_{1}, y_{2}\right)
$$


where

$$
\begin{aligned}
& d Y_{0}(t)=d t ; \quad Y_{0}(0)=s \in \mathbb{R} \\
& d Y_{1}(t)=d V^{(\pi)}(t)=Y_{1}\left(t^{-}\right)\left[\alpha \pi(t) d t+\pi\left(t^{-}\right) \int_{\mathbb{R}} z \tilde{N}(d t, d z)\right] ; \quad Y_{1}(0)>0 \\
& d Y_{2}(t)=-Y_{2}\left(t^{-}\right) \int_{\mathbb{R}} \theta_{1}(t, z) \tilde{N}(d t, d z) ; \quad Y_{2}(0)=y_{2}>0 .
\end{aligned}
$$

The generator of $Y(\cdot)$ then becomes (see (3.9))

$$
\begin{aligned}
A^{\theta_{1}, \pi} & \varphi\left(s, y_{1}, y_{2}\right)=\frac{\partial \varphi}{\partial s}+y_{1} \alpha \pi \frac{\partial \varphi}{\partial y_{1}} \\
& +\int_{\mathbb{R}}\left\{\varphi\left(s, y_{1}+y_{1} \pi z, y_{2}-y_{2} \theta_{1}(z)\right)-\varphi\left(s, y_{1}, y_{2}\right)\right. \\
& \left.-y_{1} \pi z \frac{\partial \varphi}{\partial y_{1}}+y_{2} \theta_{1}(z) \frac{\partial \varphi}{\partial y_{2}}\right\} \nu(d z) .
\end{aligned}
$$

Therefore, to solve the corresponding HJBI equation, we first fix $\pi \in K_{2}$ and minimize

$$
\begin{gathered}
h\left(\theta_{1}(\cdot)\right):=\int_{\mathbb{R}}\left\{\varphi\left(s, y_{1}+y_{1} \pi z, y_{2}-y_{2} \theta_{1}(z)\right)-\varphi\left(s, y_{1}, y_{2}\right)\right. \\
\left.-y_{1} \pi z \frac{\partial \varphi}{\partial y_{1}}+y_{2} \theta_{1}(z) \frac{\partial \varphi}{\partial y_{2}}\right\} \nu(d z)
\end{gathered}
$$

over all functions $\theta_{1}(z)$.

We can minimize this pointwise for each $z$ and get a minimum point

$$
\hat{\theta}_{1}(z)=\hat{\theta}_{1}(y, z) \in \operatorname{Argmin} \psi\left(\theta_{1}\right)
$$

where

$$
\psi\left(\theta_{1}\right)=\varphi\left(s, y_{1}(1+\pi z), y_{2}\left(1-\theta_{1}\right)\right)+y_{2} \theta_{1} \frac{\partial \varphi}{\partial y_{2}} .
$$

The first order condition for a minimum $\hat{\theta}_{1}$ of $\psi$ is

$$
\frac{\partial \varphi}{\partial y_{2}}\left(s, y_{1}(1+\pi z), y_{2}\left(1-\hat{\theta}_{1}\right)\right)=\frac{\partial \varphi}{\partial y_{2}}\left(s, y_{1}, y_{2}\right) .
$$

This value $\hat{\theta}_{1}=\hat{\theta}_{1}(y, z, \pi)$ is substituted into (4.26), and the resulting expression

$$
\begin{aligned}
k(\pi) & :=y_{1} \alpha \pi \frac{\partial \varphi}{\partial y_{1}}+\int_{\mathbb{R}}\left\{\varphi\left(s, y_{1}(1+\pi z), y_{2}\left(1-\hat{\theta}_{1}(s, z, \pi)\right)\right)\right. \\
& \left.-y_{1} \pi z \frac{\partial \varphi}{\partial y_{1}}+y_{2} \hat{\theta}(y, z, \pi) \frac{\partial \varphi}{\partial y_{2}}\right\} \nu(d z)
\end{aligned}
$$


is then maximized with respect to $\pi$. Call this maximum point $\hat{\pi}=\hat{\pi}(y)$.

Then the pair $\left(\hat{\theta}_{1}(y, z, \hat{\pi}(y)), \hat{\pi}(y)\right)$ is our candidate for the optimal control, and our candidate $\varphi(y)$ for the value function $\Phi(y)$ is the solution of the corresponding integro-differential equation

$$
\begin{aligned}
\frac{\partial \varphi}{\partial s}+ & y_{1} \alpha \hat{\pi}(y) \frac{\partial \varphi}{\partial y_{1}}+\int_{\mathbb{R}}\left\{\varphi\left(s, y_{1}(1+\hat{\pi}(y) z), y_{2}\left(1-\hat{\theta}_{1}(y, z, \hat{\pi}(y))\right)\right)\right. \\
- & \varphi\left(s, y_{1}, y_{2}\right)-y_{1} \hat{\pi}(y) z \frac{\partial \varphi}{\partial y_{1}} \\
& \left.+y_{2} \hat{\theta}_{1}(y, z, \hat{\pi}(y)) \frac{\partial \varphi}{\partial y_{2}}\right\} \nu(d z)=0 \quad \text { for } s<T,
\end{aligned}
$$

with boundary values

$$
\varphi\left(T, y_{1}, y_{2}\right)=y_{1} y_{2}+\zeta_{0}\left(y_{2}\right) .
$$

\section{An HJBI equation for Nash equilibria of gen- eral stochastic differential games}

Let $Y(t)=Y^{u}(t)$ be a controlled jump diffusion, as described in (3.1). Let $u=(\theta, \pi)$, where $\theta=\left(\theta_{0}, \theta_{1}\right) \in \Theta$ and $\pi=\left(\pi_{0}, \pi\right) \in \Pi$ are admissible controls for player 1 and 2, respectively, as in (3.5) and (3.6). Let us now assume that there are two performance functionals of the form (with $\mathcal{S}$ and $\tau_{\mathcal{S}}$ as before)

$$
J_{i}^{u}(y)=E^{y}\left[\int_{0}^{\tau_{s}} f_{i}(Y(t), u(t)) d t+g_{i}\left(Y\left(\tau_{\mathcal{S}}\right)\right)\right] ; \quad i=1,2 .
$$

We may regard $J_{i}^{u}(y)$ as the payoff to player number $i$ if the players use the controls $\left(\theta_{0}, \theta_{1}\right)$ and $\left(\pi_{0}, \pi_{1}\right)$, respectively.

Definition 5.1 A pair $\left(\theta^{*}, \pi^{*}\right) \in \Theta \times \Pi$ is called a Nash equilibrium for the stochastic differential game (3.1), (5.1) if the following holds:

$$
\begin{aligned}
& J_{1}^{\theta, \pi^{*}}(y) \leq J_{1}^{\theta^{*}, \pi^{*}}(y) \quad \text { for all } \quad \theta \in \Theta, y \in \mathcal{S} \\
& J_{2}^{\theta^{*}, \pi}(y) \leq J_{2}^{\theta^{*}, \pi^{*}}(y) \quad \text { for all } \quad \pi \in \Pi, y \in \mathcal{S}
\end{aligned}
$$

Condition (5.2) states that if player 2 uses the control $\pi^{*}$, then it is optimal for player 1 to use the control $\theta^{*}$. Similarly, condition (5.3) states that if player 1 uses $\theta^{*}$, then it is optimal for player 2 to use $\pi^{*}$. Thus $\left(\theta^{*}, \pi^{*}\right)$ is an equilibrium point in the sense that there is no reason for each individual player to deviate from it, as long as the other player does not.

As in Section 3 we restrict ourselves to Markov controls, and we use the same notation for $A^{\theta, \pi}$ and $\mathcal{T}$ as there. The following result may be regarded as a generalization of Theorem 3.2: 


\section{Theorem 5.2 (HJBI equations for Nash equilibria)}

Suppose there exists functions $\varphi_{i} \in C^{2}(\mathcal{S}) \cap C(\overline{\mathcal{S}}) ; i=1,2$ and a Markov control $(\hat{\theta}(y), \hat{\pi}(y)) \in \Theta \times \Pi$ such that

(i) $A^{\theta, \hat{\pi}} \varphi_{1}(y)+f_{1}(y, \theta, \hat{\pi}(y))$ $\leq A^{\hat{\theta}, \hat{\pi}} \varphi_{1}(y)+f_{1}(y, \hat{\theta}(y), \hat{\pi}(y))=0$ for all $\theta \in K_{1}, y \in \mathcal{S}$

(ii) $A^{\hat{\theta}, \pi} \varphi_{2}(y)+f_{2}(y, \hat{\theta}(y), \pi)$

$$
\leq A^{\hat{\theta}, \hat{\pi}} \varphi_{2}(y)+f_{2}(y, \hat{\theta}(y), \hat{\pi}(y))=0 \text { for all } \pi \in K_{2}, y \in \mathcal{S}
$$

(iii) $A^{\theta, \pi}\left(\tau_{\mathcal{S}}\right) \in \partial \mathcal{S}$ a.s. on $\left\{\tau_{\mathcal{S}}<\infty\right\}$ and

$$
\begin{aligned}
& \lim _{t \rightarrow \tau_{\mathcal{S}}^{-}} \varphi_{i}\left(Y^{\theta, \pi}(t)\right)=g_{i}\left(Y^{\theta, \pi}\left(\tau_{\mathcal{S}}\right)\right) \chi_{\left\{\tau_{\mathcal{S}}<\infty\right\}} \text { a.s. for } i=1,2 \text { and for all } \\
& (\theta, \pi) \in \Theta \times \Pi \text {. }
\end{aligned}
$$

(iv) the families $\left\{\varphi_{i}\left(Y^{\theta, \pi}(\tau)\right)\right\}_{\tau \in \mathcal{T}}$ are uniformly integrable for all $y \in \mathcal{S}$, $(\theta, \pi) \in \Theta \times \Pi, i=1,2$.

Then $(\hat{\theta}, \hat{\pi})$ is a Nash equilibrium for the game (3.1), (5.1) and

$$
\begin{aligned}
& \varphi_{1}(y)=\sup _{\theta \in \Theta} J_{1}^{\theta, \hat{\pi}}(y)=J_{1}^{\hat{\theta}, \hat{\pi}}(y) \\
& \varphi_{2}(y)=\sup _{\pi \in \Pi} J_{2}^{\hat{\theta}, \pi}(y)=J_{2}^{\hat{\theta}, \hat{\pi}}(y) .
\end{aligned}
$$

Proof. We proceed as in the proof of Theorem 3.2. With $Y=Y^{\theta, \hat{\pi}}(y)$ we get by the Dynkin formula, using (i),

$$
\begin{gathered}
E^{y}\left[\varphi_{1}\left(Y\left(\tau_{\mathcal{S}}^{(N)}\right)\right)\right]=\varphi(y)+E^{y}\left[\int_{0}^{\tau_{\mathcal{S}}^{(N)}} A^{\theta, \hat{\pi}} \varphi_{1}(Y(t)) d t\right] \\
\leq \varphi_{1}(y)-E^{y}\left[\int_{0}^{\tau_{\mathcal{S}}^{(N)}} f_{1}(Y(t), \theta(Y(t)), \hat{\pi}(Y(t)) d t] .\right.
\end{gathered}
$$

Hence

$$
\varphi_{1}(y) \geq E^{y}\left[\int_{0}^{\tau_{\mathcal{S}}^{(N)}} f_{1}(Y(t), \theta(Y(t)), \hat{\pi}(Y(t))) d t+\varphi_{1}\left(Y\left(\tau_{\mathcal{S}}^{(N)}\right)\right)\right]
$$

Letting $N \rightarrow \infty$ we obtain

$$
\varphi_{1}(y) \geq J_{1}^{\theta, \hat{\pi}}(y) .
$$

Since this holds for all $\theta \in \Theta$ we have

$$
\varphi_{1}(y) \geq \sup _{\theta \in \Theta} J_{1}^{\theta, \hat{\pi}}(y) .
$$


Similarly, applying the argument above to the control $(\hat{\theta}, \hat{\pi})$ we get equality in (5.6), i.e.

$$
\varphi_{1}(y)=J_{1}^{\hat{\theta}, \hat{\pi}}(y) .
$$

Combining (5.6) and (5.7) we get

$$
\varphi_{1}(y)=\sup _{\theta \in \Theta} J_{1}^{\theta, \hat{\pi}}(y)=J_{1}^{\hat{\theta}, \hat{\pi}}(y),
$$

which is (5.4).

Statement (5.5) is proved in the same way. These two statements imply that $(\hat{\theta}, \hat{\pi})$ is a Nash equilibrium and the proof is complete.

Example 5.3 To illustrate Theorem 5.2 we use it to solve a jump diffusion version of a problem which was solved in [24] in the continuous diffusion case (by a different method):

Suppose the states $X_{1}(t), X_{2}(t)$ of two companies at time $t$ are described by

$$
\left\{\begin{array}{l}
d X_{1}(t)=\theta(t) d t+\sigma_{11} X_{1}(t) d \eta_{1}(t)+\sigma_{12} X_{1}(t) d \eta_{2}(t) \\
X_{1}(0)=x_{1} \in \mathbb{R}
\end{array}\right.
$$

and

$$
\left\{\begin{array}{l}
d X_{2}(t)=\pi(t) d t+\sigma_{21} X_{2}(t) d \eta_{1}(t)+\sigma_{22} X_{2}(t) d \eta_{2}(t) \\
X_{2}(0)=x_{2} \in \mathbb{R}
\end{array}\right.
$$

Here $\theta(t), \pi(t)$ are the controls to be used by company 1 and 2 , respectively. We assume that $\sigma_{i j}$ are constants and $\eta_{i}(t)=\int_{0}^{t} \int_{\mathbb{R}} z \tilde{N}_{i}(d s, d z)$ are independent pure jump Lévy martingales.

The payoff functionals to company 1 and company 2 are assumed to have the form

$$
J_{1}^{\theta, \pi}\left(s, x_{1}, x_{2}\right)=E^{x_{1}, x_{2}}\left[\int_{0}^{T-s}-\alpha_{1} \theta^{2}(t) X_{2}^{2}(t) d t+\gamma_{1} X_{1}^{2}(T) X_{2}^{2}(T)\right]
$$

and

$$
J_{2}^{\theta, \pi}\left(s, x_{1}, x_{2}\right)=E^{x_{1}, x_{2}}\left[\int_{0}^{T-s}-\alpha_{2} \pi^{2}(t) X_{1}^{2}(t) d t+\gamma_{2} X_{1}^{2}(T) X_{2}^{2}(T)\right],
$$

respectively. Here $\alpha_{i}$ and $\gamma_{i}$ are positive constants, $i=1,2$.

A possible interpretation of this is that $\theta(t), \pi(t)$ represent investment rates in company 1 and 2 , respectively. But for each company the size of the other 
heats up the economy in such a way that both the terminal payoff and the energy cost rate is proportional to the squared size of the other.

To find a possible Nash equilibrium $(\hat{\theta}, \hat{\pi}) \in \Theta \times \Pi$ for this game, we first note that the generator of the process $Y(t):=\left(s+t, X_{1}(t), X_{2}(t)\right)$ is given by, with $y=\left(s, x_{1}, x_{2}\right)$,

$$
\begin{aligned}
& A^{\theta, \pi} \varphi(y)=\frac{\partial \varphi}{\partial s}+\theta \frac{\partial \varphi}{\partial x_{1}}+\pi \frac{\partial \varphi}{\partial x_{2}}+\int_{\mathbb{R}}\left\{\varphi\left(s, x_{1}+x_{1} \sigma_{11} z, x_{2}+x_{2} \sigma_{21} z\right)\right. \\
& \left.\quad-\varphi\left(s, x_{1}, x_{2}\right)-x_{1} \sigma_{11} z \frac{\partial \varphi}{\partial x_{1}}-x_{2} \sigma_{21} z \frac{\partial \varphi}{\partial x_{2}}\right\} \nu_{1}(d z) \\
& +\int_{\mathbb{R}}\left\{\varphi\left(s, x_{1}+x_{1} \sigma_{12} z, x_{2}+x_{2} \sigma_{22} z\right)\right. \\
& \left.\quad-\varphi\left(s, x_{1}, x_{2}\right)-x_{1} \sigma_{12} z \frac{\partial \varphi}{\partial x_{1}}-x_{2} \sigma_{22} z \frac{\partial \varphi}{\partial x_{2}}\right\} \nu_{2}(d z)
\end{aligned}
$$

where $\nu_{i}(d z)$ are the Lévy measures of $\eta_{i}(\cdot)$, respectively, $i=1,2$.

We also note that in this problem we have

$$
f_{1}\left(s, x_{1}, x_{2}, \theta, \pi\right)=-\alpha_{1} \theta^{2} x_{2}^{2}, \quad g_{1}\left(s, x_{1}, x_{2}\right)=\gamma_{1} x_{1}^{2} x_{2}^{2}
$$

and

$$
f_{2}\left(s, x_{1}, x_{2}, \theta, \pi\right)=-\alpha_{2} \pi^{2} x_{1}^{2}, \quad g_{2}\left(s, x_{1}, x_{2}\right)=\gamma_{2} x_{1}^{2} x_{2}^{2} .
$$

We first fix $\pi \in \mathbb{R},\left(s, x_{1}, x_{2}\right) \in \mathbb{R}^{3}$ and maximize

$$
A^{\theta, \pi} \varphi_{1}\left(s, x_{1}, x_{2}\right)+f_{1}\left(s, x_{1}, x_{2}, \theta, \pi\right)
$$

with respect to $\theta$, for a given function $\varphi_{1}$ (to be determined). This amounts to maximizing

$$
h_{1}(\theta):=\frac{\theta \partial \varphi_{1}}{\partial x_{1}}-\alpha_{1} \theta^{2} x_{2}^{2} ; \quad \theta \in \mathbb{R}
$$

The maximum is attained at

$$
\theta=\hat{\theta}=\frac{1}{2} \alpha_{1}^{-1} x_{2}^{-2} \cdot \frac{\partial \varphi_{1}}{\partial x_{1}} .
$$

For this choice of $\theta$ and with $\pi=\hat{\pi}$ (to be determined) we are required to have

$$
A^{\hat{\theta}, \hat{\pi}} \varphi_{1}(y)+f_{1}(y, \hat{\theta}(y), \hat{\pi}(y))=0
$$

i.e.

$$
\begin{aligned}
& \frac{\partial \varphi_{1}}{\partial s}+\frac{1}{2} \alpha_{1}^{-1} x_{2}^{-2}\left(\frac{\partial \varphi_{1}}{\partial x_{1}}\right)^{2}+\hat{\pi} \frac{\partial \varphi_{1}}{\partial x_{2}}+\sum_{j=1}^{2} \int_{\mathbb{R}}\left\{\varphi _ { 1 } \left(s, x_{1}\right.\right. \\
& \left.+x_{1} \sigma_{1 j} z, x_{2}+x_{2} \sigma_{2 j} z\right)-\varphi_{1}\left(s, x_{1}, x_{2}\right)-x_{1} \sigma_{1 j} z \frac{\partial \varphi_{1}}{\partial x_{1}} \\
& \left.-x_{2} \sigma_{2 j} z \frac{\partial \varphi_{1}}{\partial x_{2}}\right\} \nu_{j}(d z)-\frac{1}{4} \alpha_{1}^{-1} x_{2}^{-2}\left(\frac{\partial \varphi_{1}}{\partial x_{1}}\right)^{2}=0
\end{aligned}
$$


Let us try functions $\varphi_{i}$ of the form

$$
\varphi_{i}\left(s, x_{1}, x_{2}\right)=k_{i}(s) x_{1}^{2} x_{2}^{2} ; \quad i=1,2
$$

where $k_{i}(s)$ are functions to be determined. Then

$$
\hat{\theta}=\alpha_{1}^{-1} k_{1}(s) x_{1}
$$

and (5.15) gets the form

$$
k_{1}^{\prime}(s)+\alpha_{1}^{-1} k_{1}^{2}(s)+2 k_{1}(s) \frac{\hat{\pi}}{x_{2}}+a k_{1}(s)=0,
$$

where

$$
\begin{aligned}
a=\sum_{j=1}^{2} \int_{\mathbb{R}} & z^{2}\left\{4 \sigma_{1 j} \sigma_{2 j}+\sigma_{1 j}^{2}+\sigma_{2 j}^{2}\right. \\
& \left.+z\left(2 \sigma_{1 j} \sigma_{2 j}^{2}+2 \sigma_{2 j} \sigma_{1 j}^{2}\right)+z^{2} \sigma_{1 j}^{2} \sigma_{2 j}^{2}\right\} \nu_{j}(d z) .
\end{aligned}
$$

Similarly, if we fix $\theta$ and maximize $A^{\theta, \pi} \varphi_{2}\left(s, x_{1}, x_{2}\right)+f_{2}\left(s, x_{1}, x_{2}, \theta, \pi\right)$ with respect to $\pi$ we find the maximum point

$$
\pi=\hat{\pi}=\frac{1}{2} \alpha_{2}^{-1} x_{1}^{-2} \frac{\partial \varphi_{2}}{\partial x_{2}}=\alpha_{2}^{-1} k_{2}(s) x_{2}
$$

and the requirement

$$
A^{\hat{\theta}, \hat{\pi}} \varphi_{2}(y)+f_{2}(y, \hat{\theta}(y), \hat{\pi}(y))=0
$$

leads to the equation

$$
k_{2}^{\prime}(s)+2 \alpha_{1}^{-1} k_{1}(s) k_{2}(s)+\alpha_{2}^{-1} k_{2}^{2}(s)+a k_{2}(s)=0 .
$$

Combining (5.18) and (5.20) we get the 2-dimensional Riccati equation

$$
\begin{array}{ll}
k_{1}^{\prime}(s)+\alpha_{1}^{-1} k_{1}^{2}(s)+2 \alpha_{2}^{-1} k_{1}(s) k_{2}(s)+a k_{1}(s)=0 ; & s<T \\
k_{2}^{\prime}(s)+\alpha_{2}^{-1} k_{2}^{2}(s)+2 \alpha_{1}^{-1} k_{1}(s) k_{2}(s)+a k_{2}(s)=0 ; & s<T \\
k(T)=\left[\begin{array}{l}
\gamma_{1} \\
\gamma_{2}
\end{array}\right] &
\end{array}
$$

These equations can be solved explicitly using standard methods for solving Riccati equations, on the condition that our coefficients are such that $k_{1}$ and $k_{2}$ do not explode. See e.g [29] for information about Riccati equations.

Based on Theorem 5.2 and Remark 3.3 we can summarize the above as follows: 
Theorem 5.4 Let $k(s)=\left[\begin{array}{l}k_{1}(s) \\ k_{2}(s)\end{array}\right]$ be the solution of the Riccati equation (5.21)(5.23). Then the pair $(\hat{\theta}, \hat{\pi})$ given by

$$
\hat{\theta}\left(s, x_{1}, x_{2}\right)=\alpha_{1}^{-1} k_{1}(s) x_{1}, \quad \hat{\pi}\left(s, x_{1}, x_{2}\right)=\alpha_{2}^{-1} k_{2}(s) x_{2}
$$

is a Nash equilibrium of the jump diffusion game (5.8)-(5.11). The corresponding equilibrium performances are

$$
\varphi_{i}\left(s, x_{1}, x_{2}\right)=J_{i}^{\hat{\theta}, \hat{\pi}}\left(s, x_{1}, x_{2}\right)=k_{i}(s) x_{1}^{2} x_{2}^{2} ; \quad i=1,2 .
$$

Acknowledgments This work was partially supported by the NUFU project Pro x2 66/2003. We are grateful to Mark Davis, Mrinal Ghosh, Kristoffer Haugen, Goran Peskir and Jun Sekine for helpful comments.

\section{References}

[1] D. Applebaum: Lévy Processes and Stochastic Calculus. Cambridge Univ. Press 2004.

[2] P. Artzner, F. Delbaen, J. M. Eber and D. Heath: Coherent measures of risk. Mathematical Finance 4 (1999), 203-228.

[3] G. Bordigoni, A. Matoussi and M. Schweizer: A stochastic control approach to a robust utility maximization problem. Manuscript, October 2005.

[4] F. Delbaen: Coherent risk measures on general probability spaces. In K. Sandmann and J. Schonbucher (editors): Advances in Finance and Stochastics, Essays in Honor of Dieter Sondermann. Springer 2002, pp. 1-37.

[5] L. C. Evans and P. E. Souganidis: Differential games and representation formulas for solutions of Hamilton-Jacobi-Isaacs equations. Indiana Univ. Math. J. 33 (1984), 773-797.

[6] M. Frittelli and E. R. Gianin: Putting order in risk measures. J. Banking and Finance 26 (2002), 1473-1486.

[7] W. H. Fleming and H. M. Soner: Controlled Markov Processes and Viscosity Solutions. 2nd edition. Springer 2006.

[8] H. Föllmer and A. Schied: Robust preferences and convex risk measures. In K. Sandmann and J. Schonbucher (editors): Advances in Finance and Stochastics, Essays in Honor of Dieter Sondermann. Springer 2002, pp. 3956 .

[9] H. Föllmer and A. Schied: Convex measures of risk and trading constraints. Finance Stochast. 2 (2002), 429-447. 
[10] W. H. Fleming and P. E. Souganidis: On the existence of value function of two-player, zero-sum stochastic differential games. Indiana Univ. Math. J. 38 (1989), 293-314.

[11] I. Giboa and D. Schmeidler: Maxmin expected utility with non-unique prior. J. Math. Economics 18 (1989), 141-153.

[12] A. Gundel: Robust utility maximization for complete and incomplete market models. Finance Stochast. 9 (2005), 151-176.

[13] P. Grandits and T. Rheinländer: On the minimal entropy martingale measure. Annals of Prob. 30 (2002), 1003-1038.

[14] D. Hernandez-Hernandez and A. Schied: Robust utility maximization in a stochastic factor model. Statistics and Decisions 24 (2006), 109-125.

[15] D. Hernandez-Hernandez and A. Schied: A control approach to robust utility maximization with logarithmic utility and time-consistent penalties. Stochastic Processes and their Appl. (to appear).

[16] R. Isaacs: Differential Games. Wiley 1965.

[17] E. R. Jakobsen and K. H. Karlsen: A maximum principle for semicontinuous functions applicable to integro-partial differential equations. Nonlinear Diff. Equ. Appl. 13 (2006), 1-29.

[18] H.Jasso-Fuentes: Noncooperative Continuous-Time Markov Processes. MSc Dissertation 2004, Center for Reseach and Advanced Studies of the National Polytechnic Institute, Mexico.

[19] E. Jouini, W. Schachermayer and N. Touzi: Optimal risk sharing for law invariant monetary utility functions. Manuscript, May 2005.

[20] R. Korn and O. Menkens: Worst-case scenario portfolio optimization: a new stochastic control approach. Math. Meth. Oper. Res. 62 (2005), 123140.

[21] H. Kaise and S.-J. Sheu: Differential games of inf-sup type and Isaacs equations. Applied Math. \& Optim. 52 (2005), 1-22.

[22] F. Maccheroni, M. Marinacci and A. Rustichini: Ambiguity aversion, malevolent nature and the variational representation of preferences. Preprint.

[23] B. Øksendal: Stochastic Differential Equations. 6th edition. Springer 2003.

[24] B. Øksendal and K. Reikvam: Stochastic differential games with controls - discussion of a specific example. In E. Lungu (editor): Proceedings of the Symposium on Mathematical Finance. University of Botswana 1997, pp. 74-82. 
[25] B. Øksendal and A. Sulem: Applied Stochastic Control of Jump Diffusions. Second Edition Springer 2007.

[26] S. Peng: Nonlinear expecations, nonlinear evaluations and risk measures. In M. Frittelli and W. Runggaldier (editors): Stochastic Methods in Finance Lectures. Springer LNM 1856 (2004), pp. 143-218.

[27] G. Peskir and J. Shorish: Market forces and dynamic asset pricing. Stoch. Anal. Appl. 20 (2002), 1027-1082.

[28] M.-C. Quenez: Optimal portfolio in a multiple priors model. Progress in Probability 58 (2004), 291-321.

[29] W. T. Reid: Riccati Differential Equations. Academic Press 1972.

[30] J. Sekine: Dynamic minimization of worst conditional expectation of shortfall. Mathematical Finance 14 (2004), 605-618.

[31] D. Talay and Z. Zheng: Worst case model risk management. Finance Stochast. 6 (2002), 517-537. 